Article

\title{
Research on the Rapid and Accurate Positioning and Orientation Approach for Land Missile-Launching Vehicle
}

\author{
Kui Li ${ }^{1,2}$, Lei Wang ${ }^{2, *}$, Yanhong Lv ${ }^{2}$, Pengyu Gao ${ }^{2}$ and Tianxiao Song ${ }^{2}$ \\ 1 School of Electronic and Information Engineering, Beihang University, Beijing 100191, China; \\ E-Mail: eric.lee_buaa@buaa.edu.cn \\ 2 School of Instrument Science and Opto-electronics Engineering, Beihang University, Beijing 100191, \\ China; E-Mails: yanhonglv@buaa.edu.cn (Y.L.); gaodiao008@163.com (P.G.); \\ songtianxiao_buaa@126.com (T.S.) \\ * Author to whom correspondence should be addressed; E-Mail: 351722wang@163.com; \\ Tel.: +86-10-8233-8337.
}

Academic Editor: Gert F. Trommer

Received: 17 July 2015 / Accepted: 15 October 2015 / Published: 20 October 2015

\begin{abstract}
Getting a land vehicle's accurate position, azimuth and attitude rapidly is significant for vehicle based weapons' combat effectiveness. In this paper, a new approach to acquire vehicle's accurate position and orientation is proposed. It uses biaxial optical detection platform (BODP) to aim at and lock in no less than three pre-set cooperative targets, whose accurate positions are measured beforehand. Then, it calculates the vehicle's accurate position, azimuth and attitudes by the rough position and orientation provided by vehicle based navigation systems and no less than three couples of azimuth and pitch angles measured by BODP. The proposed approach does not depend on Global Navigation Satellite System (GNSS), thus it is autonomous and difficult to interfere. Meanwhile, it only needs a rough position and orientation as algorithm's iterative initial value, consequently, it does not have high performance requirement for Inertial Navigation System (INS), odometer and other vehicle based navigation systems, even in high precise applications. This paper described the system's working procedure, presented theoretical deviation of the algorithm, and then verified its effectiveness through simulation and vehicle experiments. The simulation and experimental results indicate that the proposed approach can achieve positioning and orientation accuracy of $0.2 \mathrm{~m}$ and 20 " respectively in less than $3 \mathrm{~min}$.
\end{abstract}


Keywords: land vehicle; positioning and orientation; biaxial optical detection platform

\section{Introduction}

Modern war is having increasingly higher requirements for weapon launching systems. To improve the weapon's survival capability in the battlefield, it needs weapon launching systems not only to be capable of accurate aiming at the objects, but also to be able to quickly respond and to have high flexibility, agility, and reliability [1]. This means that a weapon launching system must be capable of acquiring its accurate position, attitudes and orientation rapidly [2]. Consequently, designing an approach which can obtain the accurate launching reference information rapidly for launch of weapons is of great significance and value for improvement of ground artillery's survival capability, rapid response capability and maneuvering capability.

To achieve this goal, lots of work has been done in the past. Theodolite, Inertial Navigation System (INS), odometer, land markers correct, zero-velocity correct, Global Navigation Satellite System (GNSS) are the most commonly used methods and equipment [3-6]. Some multiple integrated approaches such as INS/GNSS integration, INS and odometer integration (INS/DR) are also studied and applied [7-9].

Theodolite can provide high performance orientation information by itself [3], but its operation is usually laborious and needs relatively long time, thus it is not beneficial for battlefield survival. INS is autonomous, which does not need external measuring information and has strong anti-interference ability [10]. However, INS's positioning and orientation errors increase with time [11]. If INS has been working for a relatively long time, its reference information is hard to satisfy accurate launching requirements. GNSS can achieve high positioning results, and its navigation errors do not divergent with time, but during wartime, it has the risk of being interfered and cannot provide high precision orientation and attitude reference [12]. Integration of INS and GNSS can combine both systems' advantages [13,14]. However, it would lose some autonomy since GNSS is involved in real-time. Odometer is used to measure speed and distance of the vehicle moving on the ground. It cannot be used independently for positioning but can be used with INS for Dead Reckoning (DR) [15]. INS/DR integration approach is widely used in land weapon launching applications since it can provide relatively high precision reference information as well as keep autonomy [16]. However, for most approaches mentioned above, if precise orientation and attitudes are needed, high performance INS is necessary, which is always expensive and needs much preparation time.

Based on this background, the paper proposed a new solution. The approach only needs land vehicle based INS or INS/DR to provide rough position and orientation as iterative initial value, thus it does not have high performance requirements even in high precision applications. The new solution uses a vehicle based BODP to aim at and lock no less than three pre-set cooperative targets. Since the cooperative targets are set beforehand, their accurate coordinates can be measured by differential GNSS (DGNSS). After acquiring measurements from BODP, the vehicle's accurate position and orientation can be calculated. The proposed approach does not depend on GNSS and is autonomous. This paper described the basic principle of the system. Section 2 describes the system working procedure, and presents theoretical deviation of the algorithm. Simulations results under different conditions are given in Section 3. Vehicle experiments are 
carried out and experiment results are presented and analyzed in Section 4, and conclusions are drawn in Section 5.

\section{Principle of the Proposed Approach}

\subsection{System Working Procedure}

The vehicle positioning and orientation system contains several subsystems, including INS/DR integration system, a vehicle based BODP, and several cooperative targets arranged around the launching areas. The vehicle starts from home and stops at any point and to any direction in the selected launching areas. The system uses the targets' coordinates and vehicle's coordinates provided by INS/DR to calculate targets' rough azimuth and pitch angle relative to vehicle. Then, it rotates BODP to search and lock the targets, and output the targets' precise azimuth and pitch angles relative to vehicle. After acquiring no less than three couples of azimuth and pitch angles, the system could calculate vehicle's accurate horizontal position and orientation, and then, the vehicle's accurate height and attitudes. The following section would give detailed positioning, orientation and attitudes determination algorithms that proposed.

\subsection{Orientation and Horizontal Position Determination Algorithm}

Establish a rectangular coordinate system shown in Figure $1 \mathrm{~A}-\mathrm{C}$ are cooperative targets, and $\mathrm{O}$ is the vehicle's parking position. Use $\left(x_{t m i}, y_{t m i}, z_{t m i}\right), \mathrm{i}=1,2,3$ to denote the targets' coordinates which are measured by DGNSS, and $\left(x_{t r i}, y_{t r i}, z_{t r i}\right) \mathrm{i}=1,2,3$ to denote the targets' true coordinates. The vehicle based INS/DR provides vehicle's coordinates as $\left(x_{0}, y_{0}, z_{0}\right)$, while the vehicle's real coordinates are $\left(x_{r}, y_{r}, z_{r}\right)$. The direction $O N$ denotes true north of geographic coordinate system, and $O N^{\prime}$ denotes north direction given by INS/DR. Use $\delta \psi$ to denote the difference between $O N$ and $O N^{\prime}$. The true attitude and azimuth of the vehicle are $\theta_{\mathrm{r}}, \gamma_{\mathrm{r}}, \psi_{\mathrm{r}}$ respectively, and $\theta, \gamma, \psi$ are vehicle's attitude and azimuth provided by INS/DR. The BODP aiming at the targets A, B, C and output three couples of azimuth and pitch angles denoted by $\left(\psi_{t m 1}, \phi_{m 1}\right),\left(\psi_{t m 2}, \phi_{m 2}\right)$ and $\left(\psi_{t m 3}, \phi_{m 3}\right)$, while the true angles are denoted by $\left(\psi_{t r 1}, \phi_{r 1}\right)$, $\left(\psi_{t r 2}, \phi_{r 2}\right)$ and $\left(\psi_{t r 3}, \phi_{r 3}\right)$.

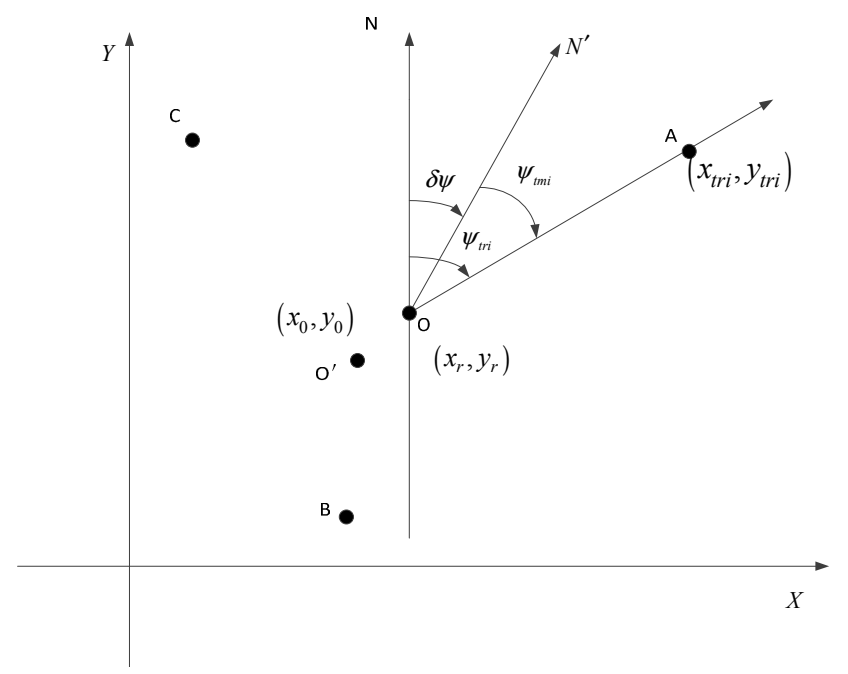

Figure 1. Orientation and horizontal position determination diagram. 
From geometric relations shown in Figure 1, we can get the following equation:

$$
\left(x_{t r i}-x_{r}\right) \cos \psi_{t r i}=\left(y_{t r i}-y_{r}\right) \sin \psi_{t r i}, \mathrm{i}=1,2,3
$$

Linearize Equation (1) by Taylor expansion at $\left(x_{0}, y_{0}, x_{t m i}, y_{t m i}\right)$ as follows:

$$
\begin{aligned}
\psi_{t r i}= & \psi_{t m i}+\delta \psi \\
= & \left.\psi_{t r i}\right|_{\left(x_{0}, y_{0}, x_{t m i}, y_{t m i}\right)}+\left.\frac{\partial \psi_{t r i}}{\partial x_{r}}\right|_{\left(x_{0}, y_{0}, x_{t m i}, y_{t m i}\right)} \delta x_{0}+\left.\frac{\partial \psi_{t r i}}{\partial y_{r}}\right|_{\left(x_{0}, y_{0}, x_{t m i}, y_{t m i}\right)} \delta y_{0}, \mathrm{i}=1,2,3 \\
& +\left.\frac{\partial \psi_{t r i}}{\partial x_{t r i}}\right|_{\left(x_{0}, y_{0}, x_{t m i}, y_{t m i}\right)} \delta x_{t m i}+\left.\frac{\partial \psi_{t r i}}{\partial y_{t r i}}\right|_{\left(x_{0}, y_{0}, x_{t m i}, y_{t m i}\right)} \delta y_{t m i}
\end{aligned}
$$

where $\left.\psi_{t r i}\right|_{\left(x_{0}, y_{0}, x_{t m i}, y_{t m i}\right)}$ is the value of $\psi_{t r i}$ at $\left(x_{0}, y_{0}, x_{t m i}, y_{t m i}\right), \mathrm{i}=1,2,3$, and

$$
\begin{aligned}
& \frac{\partial \psi_{t r i}}{\partial x_{r}}=\frac{-1}{\left(y_{t r i}-y_{r}\right)+\left(x_{t r i}-x_{r}\right) \tan \psi_{t r i}} \frac{\partial \psi_{t r i}}{\partial y_{r}}=\frac{1}{\left(x_{t r i}-x_{r}\right)+\left(y_{t r i}-y_{r}\right) \cot \psi_{t r i}} \\
& \frac{\partial \psi_{t r i}}{\partial x_{t r i}}=\frac{1}{\left(y_{t r i}-y_{r}\right)+\left(x_{t r i}-x_{r}\right) \tan \psi_{t r i}} \frac{\partial \psi_{t r i}}{\partial y_{t r i}}=\frac{-1}{\left(x_{t r i}-x_{r}\right)+\left(y_{t r i}-y_{r}\right) \cot \psi_{t r i}}
\end{aligned}
$$

Since coordinates of the cooperative targets $\mathrm{A}, \mathrm{B}$ and $\mathrm{C}$ are acquired by precise measurement, compared to $\delta x_{0}$ and $\delta y_{0}, \delta x_{t m i}$ and $\delta y_{t m i}$ can be ignored. Then, Equation (2) can be simplified as follows:

$$
\psi_{t m i}+\left.\delta \psi \doteq \psi_{t r i}\right|_{\left(x_{0}, y_{0}, x_{t m i}, y_{t m i}\right)}+\left.\frac{\partial \psi_{t r i}}{\partial x_{r}}\right|_{\left(x_{0}, y_{0}, x_{t m i}, y_{t m i}\right)} \delta x_{0}+\left.\frac{\partial \psi_{t r i}}{\partial y_{r}}\right|_{\left(x_{0}, y_{0}, x_{t m i}, y_{t m i}\right)} \delta y_{0}, \mathrm{i}=1,2,3
$$

If three cooperative targets are measured, the corresponding equations can be written as following:

$$
\Delta L_{H}=H_{H} \Delta E_{H}, \mathrm{i}=1,2,3
$$

where

$$
\Delta L_{H}=\left(\begin{array}{l}
\psi_{t m 1}-\left.\psi_{t r 1}\right|_{\left(x_{0}, y_{0}, x_{m 1}, y_{t m 1}\right)} \\
\psi_{t m 2}-\left.\psi_{t r 2}\right|_{\left(x_{0}, y_{0}, x_{m 2}, y_{t m 2}\right)} \\
\psi_{t m 3}-\left.\psi_{t r 3}\right|_{\left(x_{0}, y_{0}, x_{m m}, y_{t m 3}\right)}
\end{array}\right), \Delta E_{H}=\left(\begin{array}{c}
\delta x_{0} \\
\delta y_{0} \\
\delta \psi
\end{array}\right) \text { and } A_{H}=\left(\begin{array}{lll}
\left.\frac{\partial \psi_{t r 1}}{\partial x_{r}}\right|_{\left(x_{0}, y_{0}, x_{t m 1}, y_{t m 1}\right)} & \left.\frac{\partial \psi_{t r 1}}{\partial y_{r}}\right|_{\left(x_{0}, y_{0}, x_{t m 1}, y_{t m 1}\right)} & -1 \\
\left.\frac{\partial \psi_{t r 2}}{\partial x_{r}}\right|_{\left(x_{0}, y_{0}, x_{t m 2}, y_{t m 2}\right)} & \left.\frac{\partial \psi_{t r 2}}{\partial y_{r}}\right|_{\left(x_{0}, y_{0}, x_{t m 2}, y_{t m 2}\right)} & -1 \\
\left.\frac{\partial \psi_{t r 3}}{\partial x_{r}}\right|_{\left(x_{0}, y_{0}, x_{t m 3}, y_{m 3}\right)} & \left.\frac{\partial \psi_{t r 3}}{\partial y_{r}}\right|_{\left(x_{0}, y_{0}, x_{t m}, y_{t m 3}\right)} & -1
\end{array}\right)
$$

Then, the initial horizontal position and orientation errors can be calculated by the following Equation:

$$
\Delta E_{H}=\left(A_{H}{ }^{T} A_{H}\right)^{-1} A_{H}{ }^{T} \Delta L_{H}, \mathrm{i}=1,2,3
$$

After calculating $\Delta E_{H}$, use it to update and correct INS/DR positioning result $\left(\mathrm{x}_{0}, \mathrm{y}_{0}\right)$ and orientation error $\delta \psi$, recalculate each target's true azimuth $\psi_{t m i}$ relative to vehicle, and then use the corrected positioning and orientation results to do the same procedure, until each iteration correction $\Delta E_{H}$ is within the expected accuracy. By finishing the iteration, the horizontal position and orientation of the vehicle is accurately determined. 


\subsection{Attitudes and Height Determination Algorithm}

In the proposed approach, the vehicle's accurate attitudes and height determination algorithm is on the basis of orientation and horizontal position determination results. After acquiring the accurate horizontal position and orientation results in the previous section, their errors are assumed to be negligible when determining the vehicle's attitudes and height.

The relationship of vehicle and targets' position and angles measurements are shown in Figure 2:

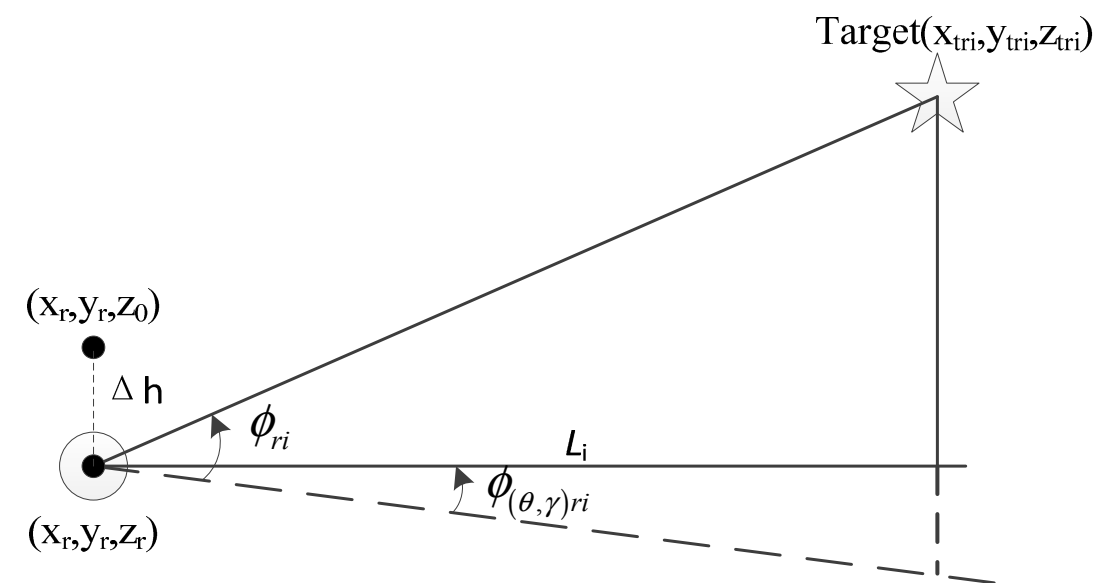

Figure 2. Relationship of vehicle and target's position and measurements.

Where $L_{i}$ is the horizontal distance between vehicle and the $i$ th target. $\phi_{(\theta, \gamma) \mathrm{r} i}$ is the true angle from optical axis of BODP when rotating around the vertical axis to the direction just below the target to the horizontal plane.

It can be seen from Figure 2 that:

$$
\tan \left(\phi_{r i}-\phi_{(\theta, \gamma) \mathrm{r} i}\right)=\frac{z_{t i}-z_{r}}{L_{i}}, \mathrm{i}=1,2,3
$$

From the Euler angles rotation relationship and Figure 2, the following equation can be derived:

$$
\sin \phi_{(\theta, \gamma) r i}=\sin \gamma_{r} \sin \psi+\cos \gamma_{r} \sin \theta_{r} \cos \psi, \mathrm{i}=1,2,3
$$

In land vehicle missile-launching applications, usually relatively firm and flat areas are selected as launching point, and the attitudes of the vehicle are usually small angles. If attitudes errors provided by INS/DR are $\Delta \theta$ and $\Delta \gamma$, that is, $\theta_{r}=\theta+\Delta \theta, \gamma_{r}=\gamma+\Delta \gamma$, then Equation (7) can be simplified as:

$$
\begin{aligned}
& \phi_{(\theta, \gamma) r i}=\sin (\gamma+\Delta \gamma) \sin \psi+\cos (\gamma+\Delta \gamma) \sin (\theta+\Delta \theta) \cos \psi, \mathrm{i}=1,2,3 \\
& \doteq \theta \cos \psi_{i}+\gamma \sin \psi_{i}+\Delta \theta \cos \psi_{i}+\Delta \gamma \sin \psi_{i}
\end{aligned}
$$

The BODP measured $\phi_{(\theta, \gamma) \mathrm{r} i}$ is denoted by $\phi_{(\theta, \gamma) m i}$, which is given by:

$$
\phi_{(\theta, \gamma) m i}=\theta \cos \psi_{i}+\gamma \sin \psi_{i}, \mathrm{i}=1,2,3
$$

Use $\Delta \phi_{(\theta, \gamma) i}$ to denote error of $\phi_{(\theta, \gamma) \mathrm{r} i}$, and $\Delta \phi_{(\theta, \gamma) i}$ is given by:

$$
\Delta \phi_{(\theta, \gamma) i}=\Delta \theta \cos \psi_{i}+\Delta \gamma \sin \psi_{i}, \mathrm{i}=1,2,3
$$


From Equations (8)-(10), it can be seen that:

$$
\phi_{(\theta, \gamma) r i}=\phi_{(\theta, \gamma) m i}+\Delta \phi_{(\theta, \gamma) i}, \mathrm{i}=1,2,3
$$

Assume $\phi_{i}=\phi_{r i}-\phi_{(\theta, \gamma) m i}$, where the pitch angle $\phi_{r i}$ is measured by BODP, then the following equation can be derived:

$$
\tan \left(\phi_{r i}-\phi_{(\theta, \gamma) r i}\right)=\tan \left(\phi_{i}-\Delta \phi_{(\theta, \gamma) i}\right)=\frac{\tan \phi_{i}-\tan \Delta \phi_{(\theta, \gamma) i}}{1+\tan \phi_{i} \tan \Delta \phi_{(\theta, \gamma) i}}=\frac{z_{t i}-z_{0}}{L_{i}}+\frac{\Delta h}{L_{i}}, \mathrm{i}=1,2,3
$$

Since $\Delta \phi_{(\theta, \gamma) i}$ is small angle, $\tan \Delta \phi_{(\theta, \gamma) i} \doteq \Delta \phi_{(\theta, \gamma) i}=\Delta \theta \cos \psi_{i}+\Delta \gamma \sin \psi_{i}$, thus the following equation can be derived:

$$
\tan \phi_{i}-\frac{z_{t i}-z_{0}}{L_{i}}=\frac{\Delta h}{L_{i}}+\Delta \theta\left(1+\frac{z_{t i}-z_{0}}{L_{i}} \tan \phi_{i}\right) \cos \psi_{i}+\Delta \gamma\left(1+\frac{z_{t i}-z_{0}}{L_{i}} \tan \phi_{i}\right) \sin \psi_{i}, \mathrm{i}=1,2,3
$$

If three cooperative targets are measured, the corresponding equations can be written as following:

$$
\Delta L_{V}=A_{V} \Delta E_{V}, \mathrm{i}=1,2,3
$$

where,

$$
\begin{gathered}
\Delta L_{V}=\left[\tan \phi_{1}-\frac{z_{t 1}-z_{0}}{L_{1}} \tan \phi_{2}-\frac{z_{t 2}-z_{0}}{L_{2}} \tan \phi_{3}-\frac{z_{t 3}-z_{0}}{L_{3}}\right]^{T} \\
A_{V}=\left[\begin{array}{ll}
\left(1+\frac{z_{t 1}-z_{0}}{L_{1}} \tan \phi_{1}\right) \cos \psi_{1} & \left(1+\frac{z_{t 1}-z_{0}}{L_{1}} \tan \phi_{1}\right) \sin \psi_{1} \frac{1}{L_{1}} \\
\left(1+\frac{z_{t 2}-z_{0}}{L_{2}} \tan \phi_{2}\right) \cos \psi_{2} & \left(1+\frac{z_{t 2}-z_{0}}{L_{2}} \tan \phi_{2}\right) \sin \psi_{2} \frac{1}{L_{2}} \\
\left(1+\frac{z_{t 3}-z_{0}}{L_{3}} \tan \phi_{3}\right) \cos \psi_{3} & \left(1+\frac{z_{t 3}-z_{0}}{L_{3}} \tan \phi_{3}\right) \sin \psi_{3} \frac{1}{L_{3}}
\end{array}\right] \\
\Delta E_{V}=\left[\begin{array}{lll}
\Delta \theta & \Delta \gamma & \Delta h
\end{array}\right]^{T}
\end{gathered}
$$

Then, the initial height and attitudes errors can be calculated by the following equation:

$$
\Delta E_{V}=\left(A_{V}{ }^{T} A_{V}\right)^{-1} A_{V}{ }^{T} \Delta L_{V}, \mathrm{i}=1,2,3
$$

After calculating $\Delta E_{V}$, use it to update and correct INS/DR height and attitudes errors. And then use the corrected results to do the same procedure, until each iteration correction $\Delta E_{V}$ is within the expected accuracy. By finishing the iteration, the vehicle's height and attitudes can be accurately determined.

\section{Simulation Results}

\subsection{Orientation and Horizontal Position Determination Simulation}

Consider the orientation and horizontal position determination algorithm simulation first. Establish the measurement rectangular coordinate system as shown in Figure 3. Assume that the true horizontal coordinates of three targets and the vehicle are (3000 m, $200 \mathrm{~m}),(-200 \mathrm{~m},-4000 \mathrm{~m}),(-1000 \mathrm{~m}, 3000 \mathrm{~m})$ and $(-100 \mathrm{~m}, 150 \mathrm{~m})$ respectively. 


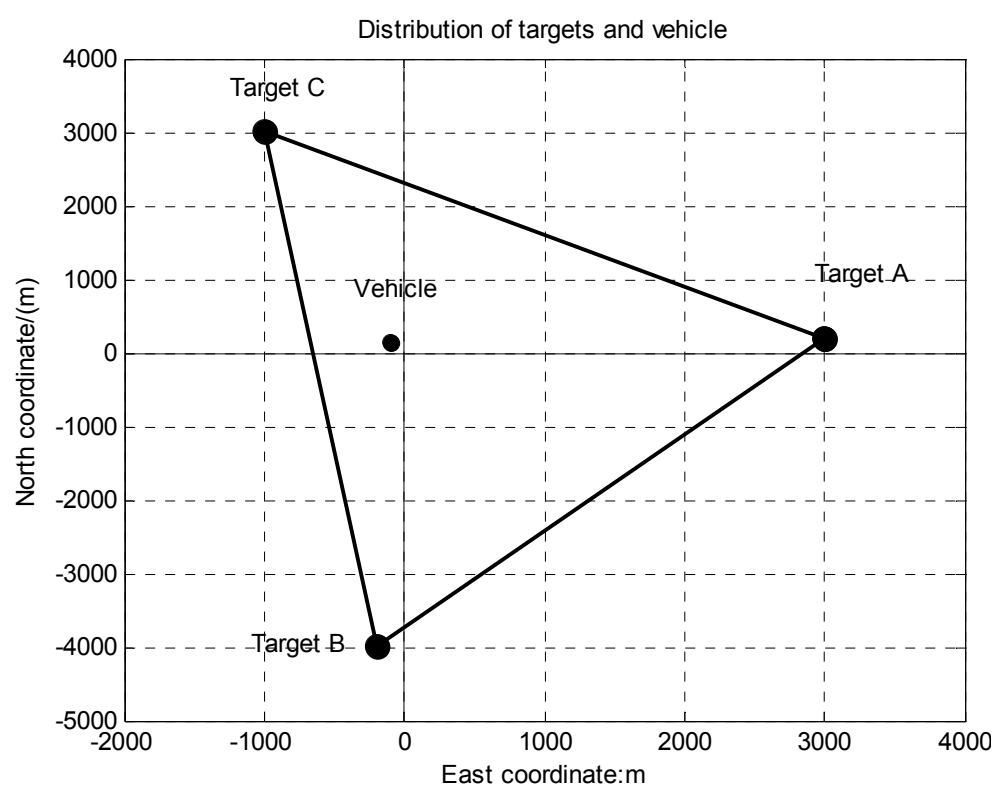

Figure 3. Distribution of targets and vehicle.

Since cooperative targets are set beforehand, their coordinates can be carefully calibrated. In the following simulation, the cooperative targets' coordinates measuring errors are assumed to be $0.3 \mathrm{~m}$ $(1 \sigma)$. Positioning error and azimuth error of vehicle based INS/DR are assumed to be $300 \mathrm{~m}(1 \sigma)$ and $0.5^{\circ}(1 \sigma)$. BODP azimuth and pitch angles measuring errors are assumed to be $5^{\prime \prime}(1 \sigma)$ when aiming at and locking the targets. Apply the proposed orientation and horizontal position determination algorithm under the above conditions and simulate 100 times. The simulation results are shown in Figures 4-6.

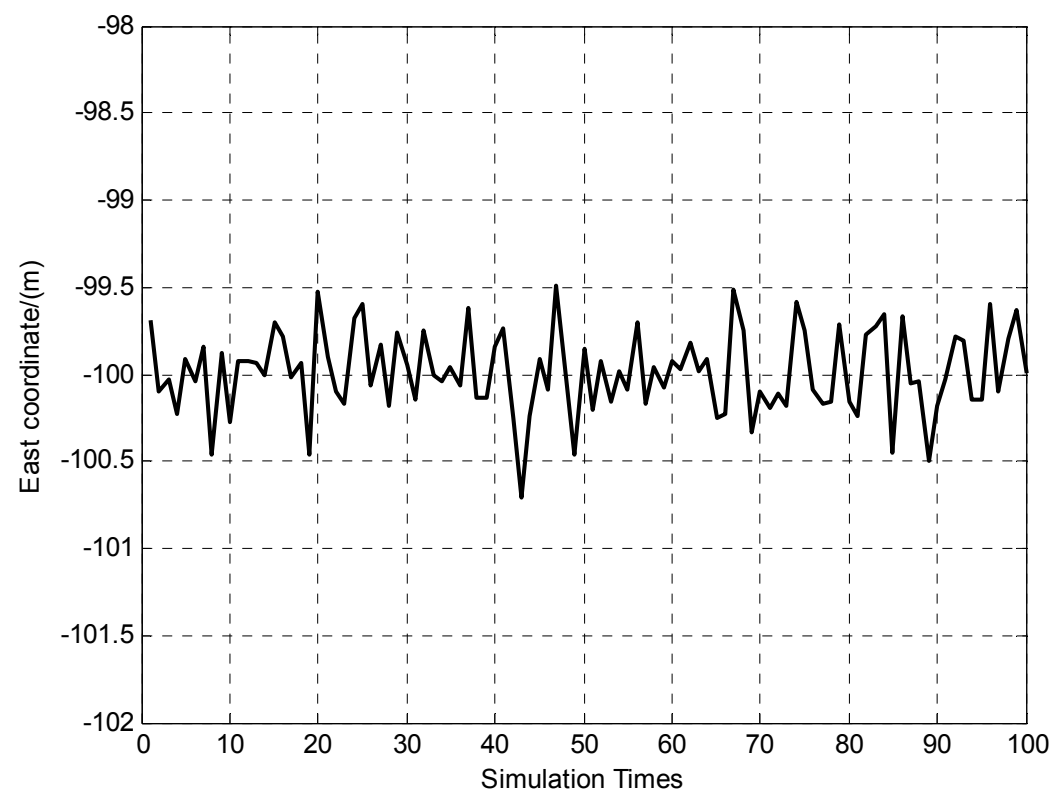

Figure 4. 100 times of east coordinate determination results. 


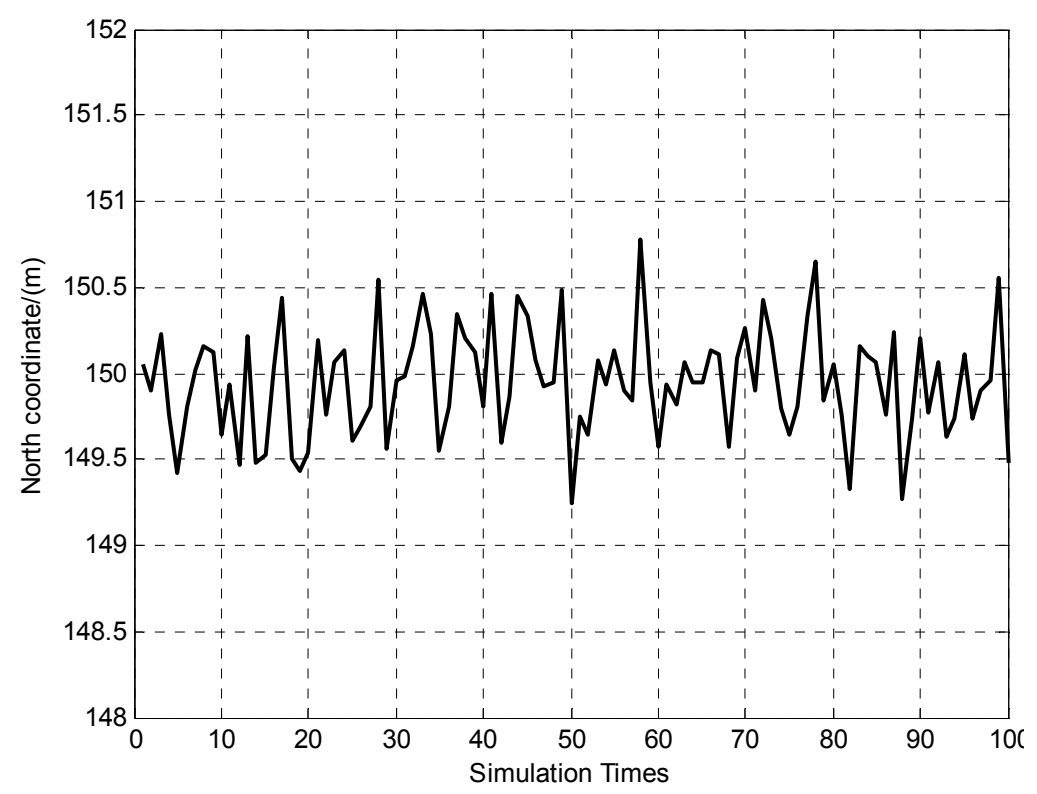

Figure 5. 100 times of north coordinate determination results.

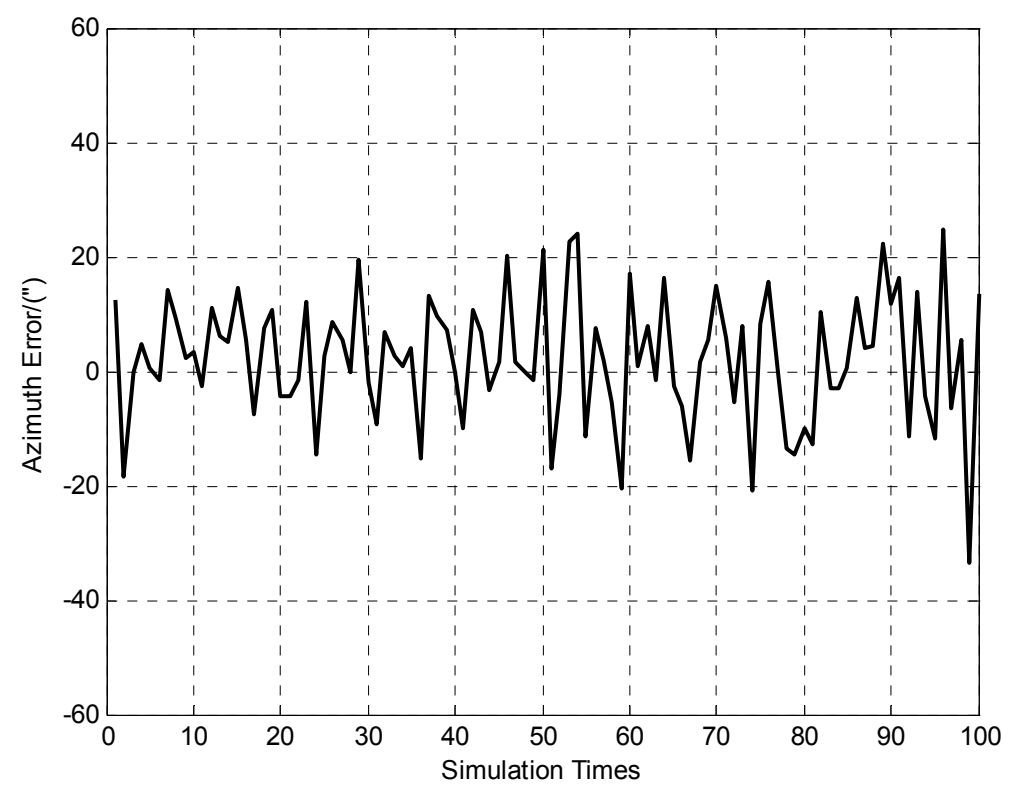

Figure 6. 100 times of azimuth error determination results.

It can be seen from Figures 4 and 5 that the positioning errors of the vehicle are within $\pm 1 \mathrm{~m}$ (Standard deviation of east and north coordinate errors are $0.2 \mathrm{~m}$ and $0.3 \mathrm{~m}$ respectively). The Figure 6 indicates that azimuth error of the vehicle is within $\pm 40 "$, and the standard deviation is 11.1".

\subsection{Attitudes and Height Determination Simulation}

Assume that the true height of the three cooperative targets and vehicle are $500 \mathrm{~m}, 480 \mathrm{~m}, 800 \mathrm{~m}$ and $60 \mathrm{~m}$ respectively. The height measuring errors of the targets are assumed to be $0.3 \mathrm{~m}(1 \sigma)$. The vehicle's true pitch and roll attitudes are $4^{\circ}$ and $3^{\circ}$, while, the attitudes error and height error of the vehicle based INS/DR are $0.2^{\circ}(1 \sigma)$ and $200 \mathrm{~m}$ respectively. In the theoretical deviation for attitudes and height determination algorithm, the vehicle's orientation and horizontal position errors were 
assumed to be zero. However, the horizontal position and orientation errors are inevitable. Consequently, in vehicle's attitudes and height determination simulations here, we add horizontal position errors of $0.3 \mathrm{~m}(1 \sigma)$ and orientation error of $20^{\prime \prime}(1 \sigma)$, which is the same accuracy as previous simulation results. Apply the proposed attitudes and height determination algorithm under the above conditions and simulate 100 times. The simulation results are shown in Figures 7-9.

It can be seen from Figures 7 and 8 that the vehicle's attitudes determination errors are within $\pm 30^{\prime \prime}$ (Standard deviation of pitch and roll errors are 7.9" and 8.8" respectively). The Figure 9 indicates that vehicle's height determination error is within $\pm 0.3 \mathrm{~m}$, and the standard deviation is $0.1 \mathrm{~m}$.

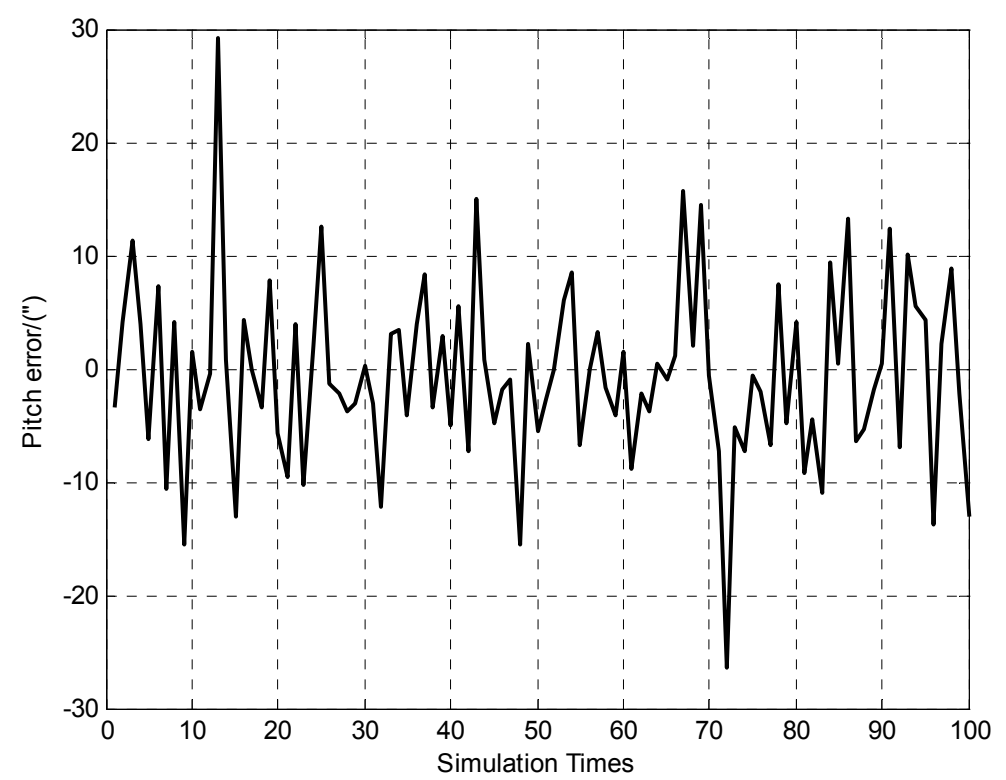

Figure 7. 100 times of pitch error determination results.

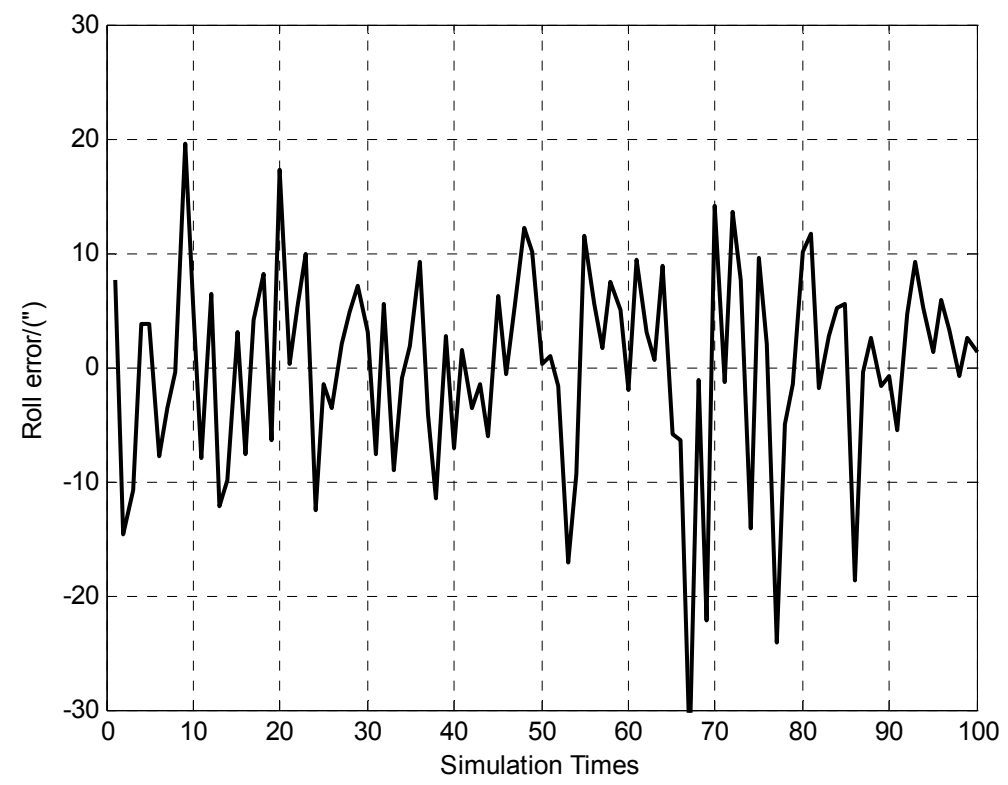

Figure 8. 100 times of roll error determination results. 


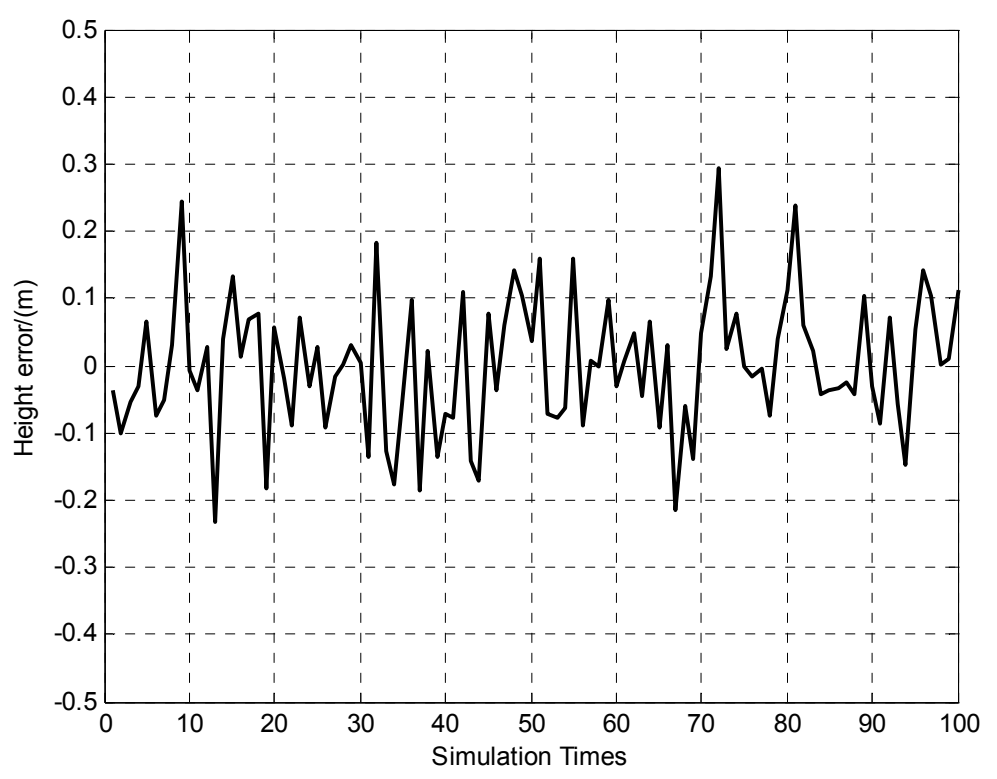

Figure 9. 100 times of height error determination results.

\section{Vehicle Experiments and Analysis}

The proposed positioning and orientation system mainly consist of vehicle based equipment and cooperative targets. The vehicle-based equipment includes INS/DR integration system, a DGNSS receiver and a BODP. The experimental vehicle and relative equipment are shown in Figure 10.

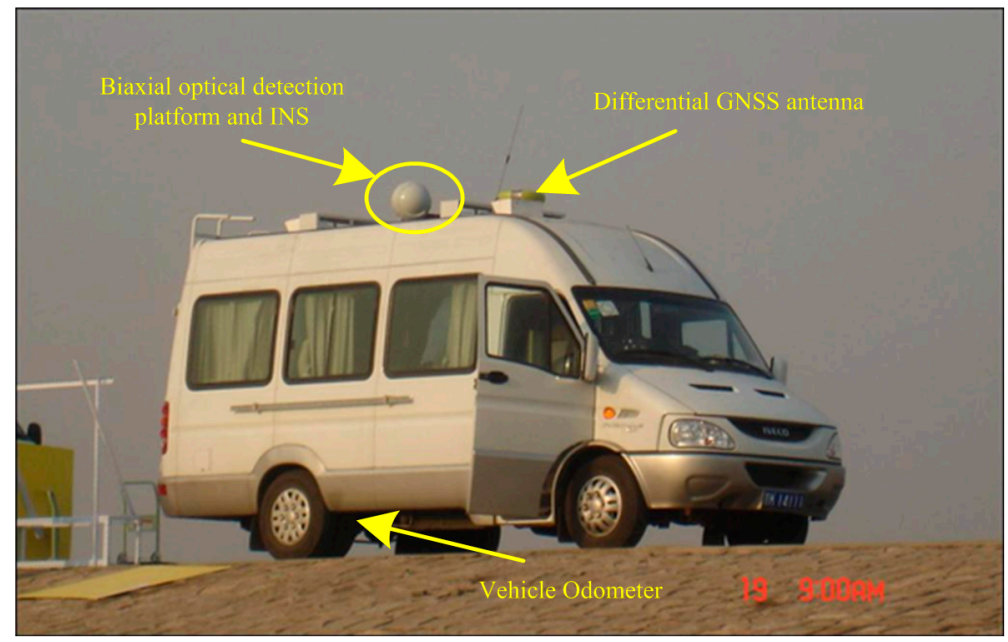

Figure 10. Experimental vehicle and relative equipment.

In the experiment, the odometer is chosen with 1024 pulse output when vehicle's wheel rounding a whole circle. And the positioning accuracy of INS/DR is about 0.001 times of distance that vehicle moves. The BODP pitch and azimuth angles measuring accuracy are about 5 " when aiming at and locking the cooperative targets. The DGNSS positioning accuracy is about $0.02 \mathrm{~m}(1 \sigma)$.

The experimental area is selected in the suburb of Beijing city. Seven cooperative targets are settled in the area, which are numbered as target no.1, 2...and 7. The distribution of the cooperative targets and vehicle parking point are shown in Figure 11. The precise coordinates of all cooperative targets are 
measured by DGNSS. In addition, the vehicle is also equipped with DGNSS to provide reference position for algorithm evaluation.

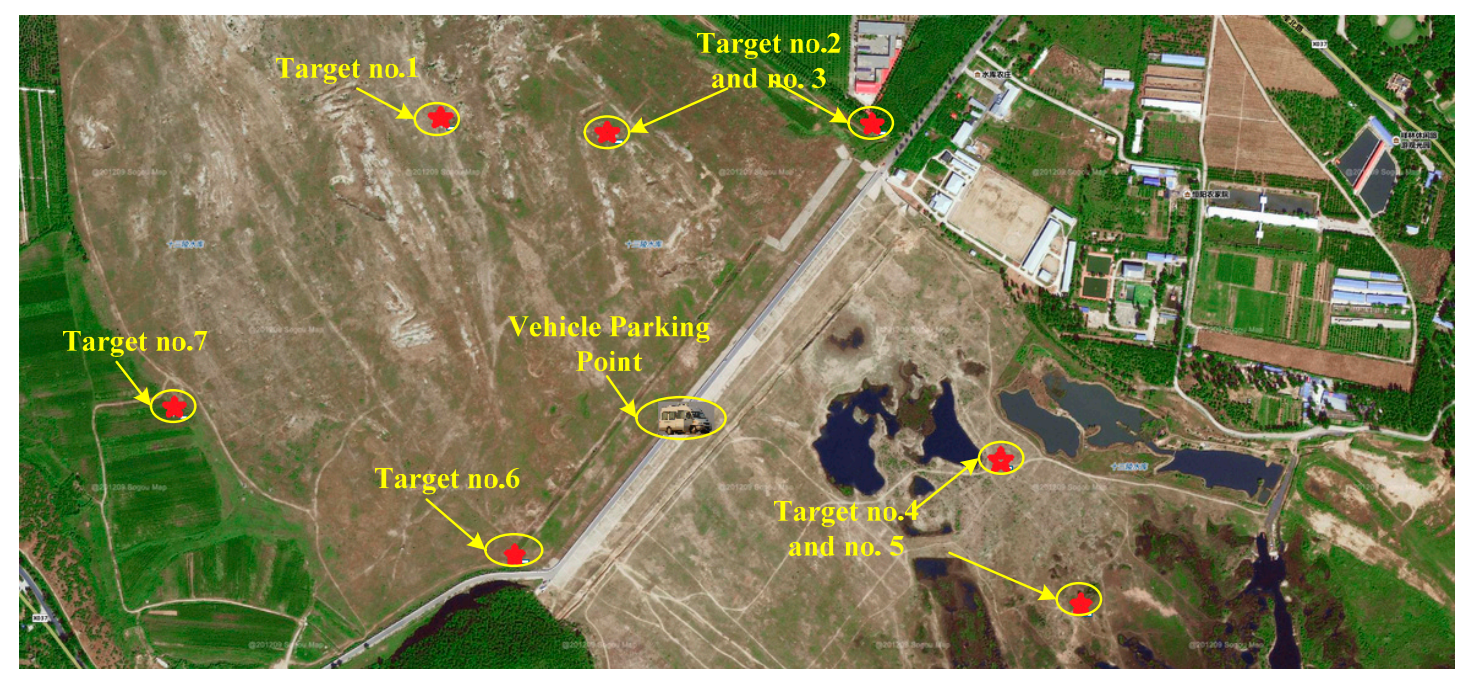

Figure 11. Distribution of the cooperative targets and vehicle.

The experimental vehicle is driven to the parking point from the starting point several kilometers away. Then, use the vehicle based DGNSS receiver to measure the vehicle's precise position as reference. The DGNSS position measuring results of the targets and the vehicle parking point are given in Table 1.

Table 1. Positions of the targets and parking points

\begin{tabular}{cccc}
\hline & Longitude $\left({ }^{\circ}\right)$ & Latitude $\left({ }^{\circ}\right)$ & Height $(\mathbf{m})$ \\
\hline Parking point & 116.2469562 & 40.2683542 & 70.90 \\
Target no.1 & 116.2428290 & 40.2722016 & 79.20 \\
Target no.2 & 116.2454469 & 40.2717759 & 79.17 \\
Target no.3 & 116.2496097 & 40.2713175 & 84.10 \\
Target no.4 & 116.2515194 & 40.2676992 & 83.89 \\
Target no.5 & 116.2546308 & 40.2661110 & 80.13 \\
Target no.6 & 116.2443096 & 40.2664876 & 102.16 \\
Target no.7 & 116.2395675 & 40.2683541 & 89.23 \\
\hline
\end{tabular}

After the BODP aims at and lock the targets, it would output azimuth and pitch angles relative to vehicle. Apply the proposed positioning and orientation approach to the equipment measurements, and then give the calculation results. In the experiment, the targets no. 2, 5, 7 were selected and the same experiment procedure was done seven times. Every time a new set of measuring parameters were acquired. Then, apply the proposed approach to seven sets of measurements and the results are given in Table 2. 
Table 2. Positioning and orientation results

\begin{tabular}{ccccccc}
\hline & Longitude $\left({ }^{\circ}\right)$ & Latitude $\left(^{\circ}\right)$ & Azimuth $\left({ }^{\circ}\right)$ & Height $(\mathbf{m})$ & Pitch $\left(^{\circ}\right)$ & Roll $\left({ }^{\circ}\right)$ \\
\hline 1 & 116.2469543 & 40.2683567 & 39.0451 & 71.10 & -0.0677 & -2.0003 \\
2 & 116.2469520 & 40.2683559 & 39.0475 & 71.08 & -0.0628 & -1.9984 \\
3 & 116.2469550 & 40.2683559 & 39.0404 & 71.19 & -0.0677 & -2.0002 \\
4 & 116.2469540 & 40.2683561 & 39.0441 & 71.20 & -0.0628 & -1.9978 \\
5 & 116.2469541 & 40.2683569 & 39.0315 & 71.06 & -0.0661 & -2.0002 \\
6 & 116.2469534 & 40.2683565 & 39.0450 & 71.92 & -0.0669 & -2.0006 \\
7 & 116.2469518 & 40.2683555 & 39.0412 & 71.03 & -0.0632 & -1.9986 \\
Mean & 116.2469535 & 40.2683562 & 39.0421 & 71.23 & -0.0653 & -1.9994 \\
Std & $0.11 \mathrm{~m}$ & $0.06 \mathrm{~m}$ & $19.0^{\prime \prime}$ & $0.31 \mathrm{~m}$ & $8.3^{\prime \prime}$ & $4.1^{\prime \prime}$ \\
\hline
\end{tabular}

It can be seen from Table 2 that in the seven experiments, the standard deviation of horizontal positioning and height errors are about $0.1 \mathrm{~m}$ and $0.3 \mathrm{~m}$, and attitudes (pitch and roll) and orientation errors are about $8 "$ and $20 "$ respectively. The statistical results indicate that the proposed approach displays good repeatability. To further verify the effectiveness of the proposed approach, five different sets of targets were selected to form different groups of measurements. The five groups of targets combinations are targets no. 2,5,7 combination, targets no. 1,4,6 combination, targets no. 3,4,7 combination, targets no. 1,3,5 combination, and targets no. 2,4,6 combination respectively. The proposed approach was applied to the five combinations of targets, and the calculation results are given in Table 3.

Table 3. Positioning and orientation results using different combination of targets

\begin{tabular}{ccccccc}
\hline Targets no. & Longitude $\left(^{\circ}\right)$ & Latitude $\left(^{\circ}\right)$ & Azimuth $\left(^{\circ}\right)$ & Height $(\mathbf{m})$ & Pitch $\left(^{\circ}\right)$ & Roll $\left(^{\circ}\right)$ \\
\hline No. 2,5,7 & 116.2469534 & 40.2683571 & 39.0409 & 71.11 & -0.0661 & -2.0000 \\
No. 1,4,6 & 116.2469549 & 40.2683563 & 39.0514 & 70.95 & -0.0715 & -2.0035 \\
No. 3,4,7 & 116.2469539 & 40.2683560 & 39.0331 & 70.93 & -0.0709 & -2.0021 \\
No. 1,3,5 & 116.2469560 & 40.2683555 & 39.0521 & 71.11 & -0.0642 & -1.9982 \\
No. 2,4,6 & 116.2469546 & 40.2683567 & 39.0310 & 70.70 & -0.0751 & -2.0063 \\
Mean & 116.2469546 & 40.2683563 & 39.0417 & 70.96 & -0.0696 & -2.0020 \\
Reference & 116.2469562 & 40.2683542 & & 70.90 & & \\
Max offset & $0.24 \mathrm{~m}$ & $0.32 \mathrm{~m}$ & $38.5^{\prime \prime}$ & $0.21 \mathrm{~m}$ & $19.8^{\prime \prime}$ & $15.5^{\prime \prime}$ \\
\hline
\end{tabular}

In Table 3, the vehicle's position provided by DGNSS is listed as reference. It can be seen that compared to the reference, the approach can achieve positioning accuracy of about $0.3 \mathrm{~m}$ maximum offset by using different sets of targets. Since there isn't any other high performance azimuth and attitude measuring equipment in the vehicle during the experiments, the true azimuth and attitude reference are not acquired. In this circumstance, the mean attitude determination results are used as reference and only repeatability when using different groups of targets are evaluated. In addition, the results show that the azimuth maximum offset relative to mean value is $38.5^{\prime \prime}$, and the maximum pitch and roll angles offset are 19.8" and 15.5", respectively.

In the verification experiments mentioned above, only three targets are selected for calculation. It takes about $40 \mathrm{~s}$ average to aim at and lock one target. If three cooperative targets are used and locked, the total time needed is about $2 \mathrm{~min}$, but if more than three targets are used, it would take more time. In 
fact, if more preparing time is allowed, the system could aim at and lock more targets, and this approach could achieve better results.

Compared with other methods listed in the introduction, the vehicle experiments' results proved that the proposed approach in this paper has many advantages. By using theodolite, it can acquire quite high orientation information $\left(20^{\prime \prime}\right)$, but the operation of theodolite requires long duration (usually more than $20 \mathrm{~min}$ ), and theodolite cannot provide position information either; however, the proposed approach in this paper is easy to operate and can obtain the same orientation accuracy as theodolite within 3 min as well as high accuracy positioning information.

The positioning accuracy of medium-accuracy of INS/DR is about several hundred meters while the orientation accuracy is about $3^{\prime}$. Compared to the INS/DR integration, the proposed approach improves the positioning and orientation accuracy significantly, with positioning error of less than $1 \mathrm{~m}$ and orientation error of about $20^{\prime \prime}$. If the same positioning and orientation accuracy were to be acquired by INS/DR, the drifts of gyros employed in INS must be less than $0.001^{\circ} / \mathrm{h}$, which will greatly increase the cost. However, the proposed approach only needs a rough position and orientation as an algorithm's iterative initial value by INS/DR, so the cost can be reduced substantially.

The positioning accuracy of GNSS can be less than $1 \mathrm{~m}$, but the GNSS signals are easy to interfere with and are requested not to be used during wartime. In addition, in order to achieve $20^{\prime \prime}$ orientation information by GNSS, multiple GNSS antennas and long base line (several decameters) are needed; it is not easy to operate in practical situation. Therefore, the proposed approach has obvious advantages in positioning and orientation accuracy, rapidity, autonomy and cost; consequently, the approach has considerable potential in rapid and accurate positioning and orientation applications for land missile-launching vehicles.

\section{Conclusions}

The paper presented a new approach to get the land vehicle's accurate position, azimuth and attitude rapidly. It uses a BODP to aim at and lock no less than three pre-set cooperative targets, whose accurate position coordinates are measured beforehand. The vehicle's accurate position and orientation through the rough position and orientation provided by vehicle based INS/DR and at least three couples of azimuth and pitch angles measured by BODP while aiming at and locking cooperative targets are then calculated. It does not have high performance requirements for vehicle-based INS. Meanwhile, it does not depend on GNSS when determining vehicle's position, attitudes and azimuth; thus, it is autonomous and difficult to interfere. The approach's effectiveness is verified and evaluated by both simulation and vehicle experiments. The results indicate that it can achieve positioning and orientation accuracy of $0.2 \mathrm{~m}$ and $20^{\prime \prime}$ respectively in $3 \mathrm{~min}$; thus, it has high potential engineering values. Still, it has lots of work to do, including the influence of targets and vehicle distribution analysis, influence of vehicle based INS/DR error on the results analysis, et al. These are the next projects for our team to accomplish in the near future. 


\section{Acknowledgments}

This research is supported and funded by the National Natural Science Foundation of China (L142200032) and long-term development strategic research of China Engineering Science and Technology (2014-zcq-01). The authors appreciate the support and funding.

\section{Author Contributions}

Kui Li and Yanhong Lv conceived and designed the study. Kui Li and Lei Wang derived and simulated the approach algorithm. Kui Li, Lei Wang, Yanhong Lv, Pengyu Gao and Tianxiao Song all participated and performed the experiments. Kui Li and Pengyu Gao wrote and edited the manuscript, Lei Wang and Tianxiao Song reviewed the manuscript. All authors read and approved the manuscript.

\section{Conflicts of Interest}

The authors declare that there are no conflicts of interest.

\section{References}

1. Kim S.B.; Lee, S.Y.; Hwang, T.H.; Choi, K.H. An advanced approach for navigation and image sensor integration for land vehicle navigation. In Proceedings of the 2014 IEEE 60th Vehicular Technology Conference, Piscataway, NJ, USA, 26-29 September 2004; pp. 4075-4078.

2. Yang, H.; Zhang, S.; Hu, Z. Modeling and parameter estimation for initial launched parameter error warship missile. Syst. Eng. Electron. 2007, 6, 931-937.

3. Li, Z. Autonomous Position and Orientation Technology for Trailer-Mounted Theodolite. Ph.D. Thesis, Changchun Institute of Optics, Fine Mechanics and Physics, Chinese Academy of Sciences, Changchun, China, 2010.

4. Alam, N.; Kealy, A.; Dempster, A.G. Cooperative Inertial Navigation for GNSS-Challenged Vehicular Environments. IEEE Trans. Intell. Transp. Syst. 2013, 8, 1370-1379.

5. Le Hieu, N.; Nguyen, V.H. Loosely coupled GPS/INS integration with Kalman filtering for land vehicle applications. In Proceedings of the 2012 International Conference on the Control, Automation and Information Sciences (ICCAIS), Ho Chi Minh City, Vietnam, 26-29 November 2012; pp. 90-95.

6. Beckmann, H.; Niedermeier, H.; Eissfeller, B. Improving GNSS road navigation integrity using MEMS INS and odometry. In Proceedings of the 2012 6th ESA Workshop on Satellite Navigation Technologies and European Workshop on GNSS Signals and Signal Processing (NAVITEC), Noordwijk, the Netherland, 5-7 December 2012; pp. 1-7.

7. Jo, K.; Chu, K.; Sunwoo, M. Interacting Multiple Model Filter-Based Sensor Fusion of GPS with In-Vehicle Sensors for Real-Time Vehicle Positioning, IEEE Transp. Intell. Trans. Syst. 2012, 3, 329-343.

8. Dumitrache, A.; Zamora, M.A.; Toledo-Moreo, R.; Skarmeta, A.G. Hybridized GPS/DR Positioning System With Unknown Initial Heading For Land Vehicles. In Proceedings of the 11th International IEEE Conference on Intelligent Transportation Systems (ITSC 2008), Beijing, China, 12-15 October 2008; pp. 974-979. 
9. Takeyama, K.; Kojima, Y.; Teramoto, E. Trajectory estimation improvement based on time-series constraint of GPS doppler and INS in urban areas. In Proceedings of the 2012 IEEE/ION Position Location and Navigation Symposium (PLANS), Myrtle Beach, SC, USA, 23-26 April 2012; pp. 700-705.

10. Park, S.K.; Suh, Y.S.; Kang, H.J.; Ro, Y.S. Zero velocity detection for inertial sensor-based personal navigation systems. In Proceedings of the SICE Annual Conference 2010, Taipei, China, 18-21 August 2010; pp. 1755-1758.

11. Li, Y. Research on the Vehicle Positioning and Orientation System Using Laser Gyro. Master's Thesis, Harbin Institute of Technology, Harbin, China, 2014.

12. Kim, S.-B.; Bazin, J.-C.; Lee, H.-K.; Choi, K.-H.; Park, S.-Y. Ground vehicle navigation in harsh urban conditions by integrating inertial navigation system, global positioning system, odometer and vision data. Radar Sonar Navig. IET 2011, 10, 814-823.

13. Amini, A.; Vaghefi, R.M.; de la Garza, J.M.; Buehrer, R.M. Improving GPS-based vehicle positioning for intelligent transportation systems. In Proceedings of the 2014 IEEE Intelligent Vehicles Symposium Proceedings, Dearborn, MI, USA, 8-11 June 2014; pp. 1023-1029.

14. Cappelle, C.; Pomorski, D.; Yang, Y. GPS/INS Data Fusion for Land Vehicle Localization. In Proceedings of the IMACS Multiconference on Computational Engineering in Systems Applications, Beijing, China, 4-6 October 2006; pp. 21-27.

15. Zhang, X.; Yang, G.; Zhang, C. Integrated navigation method for SINS and odometer. J. Beijing Univ. Aeronaut. Astronaut. 2013, 7, 922-926.

16. Georgy, J.; Noureldin, A.; Bayoumi, M. Mixture Particle Filter for Low Cost INS/Odometer/GPS Integration in Land Vehicles. In Proceedings of the Vehicular Technology Conference, 2009. VTC Spring 2009, Barcelona, Spain, 26-29 April 2009; pp. 1-5.

(C) 2015 by the authors; licensee MDPI, Basel, Switzerland. This article is an open access article distributed under the terms and conditions of the Creative Commons Attribution license (http://creativecommons.org/licenses/by/4.0/). 